\title{
船-
}

\section{Repensando la intervención psicosocial en pacientes con ictus}

\author{
Verónica Olmedo Vega \\ Trabajadora Social Sanitaria del Servicio Médico \\ de Neurología \\ Hospital Clínico Universitario de Valladolid \\ volmedo@saludcastillayleon.es \\ Ruth González Aguado \\ Trabajadora Social
}

Resumen: La realidad social está sometida a cambios constantes que exigen ajustes y reflexiones sobre cómo intervenir en ésta desde el trabajo social. Sistematizar los procesos de actuación como forma de ordenar la práctica y reflexionar sobre ella desde todas las perspectivas posibles, contribuye a ese ajuste y adaptación de los modelos de intervención, que resulten eficaces y operativos para la sociedad actual.

Por ello, se plantea el análisis de una intervención profesional con la finalidad de repensar esa forma de actuación, generar nuevas ideas y conocimientos sobre la misma y reinventar otras formas de intervención y otros 
procedimientos que contribuyan a un mayor bienestar, desde el razonamiento y la reflexión.

La experiencia seleccionada, intervención con pacientes que han sufrido un ictus, visibiliza los riesgos sociales que provoca dicha enfermedad, las consecuencias bio-psico-sociales de los mismos y la necesidad de una intervención basada en la colaboración entre diferentes disciplinas y profesiones, así como la interrelación en lo relativo a la participación de las esferas: pública, familiar, voluntaria y comunitaria.

Bajo esta premisa, procederemos a analizar las actuaciones desde el trabajo social sanitario de neurología con los pacientes de ictus, repensando las intervenciones y los modelos aplicados.

Palabras Claves: Ictus, intervención social, integral, reflexión, sistematización, rehabilitación.

\section{Rethinking psychological intervention for patients that have suffered a stroke}

Abstract: Social reality is in a state of constant flux and this requires adjustment and reflection on how to go about tackling such changes from a social work perspective. Systematizing procedures as a way of organizing praxis and reflecting on such processes from all possible angles helps adjust and adapt intervention models that might prove to be effective and workable in today's society.

Hence, analyzing professional intervention is proposed so as to rethink such a procedure, generate new ideas and knowledge about it, and reinvent different types of intervention and other procedures that contribute to greater well-being, based on reasoning and reflection.

The selected experience - treatment of patients who have suffered a stroke - draws attention to the social risks caused by the event, its biosociopsycho repercussions, and the need for collaboration among various disciplines and professions to perform such an intervention, as well as the interrelations concerning the participation of the sectors involved, i.e. public, family, community and volunteers.

Under this premise, we proceed to analyze the actions carried out by medical sociologists and social workers concerned with neurology involving stroke patients, and in so doing rethink applied models and interventions.

Keywords: Ictus, intervención social, integral, reflexión, sistematización, rehabilitación. 


\section{Repensando la intervención psicosocial en pacientes con ictus}

Verónica

Olmedo Vega

https://doi.org/10.26754/ojs_ais/ais.2019393233

Ruth

Recibido: 23/07/2017

Aceptado: 23/05/2018

González Aguado

\section{INTRODUCCIÓN}

Saber pensar es abrir los sistemas teóricos al debate, al diálogo con otras teorías, con otros pensamientos, y eso no se resuelve de manera instrumental: no basta con verificar los datos buscando encontrar en ellos la lógica de su correspondencia interna, también es necesario organizar la experiencia para dilucidar y comprender la lógica que rige el pensamiento y el tipo de necesidades presentes en su organización. Saber pensar significa pensar en el propio pensamiento, y esa capacidad de auto reflexión que hay que potenciar entre todos/as y cada uno de nosotros/as (Vélez, 2003: 21).

El trabajo social, como profesión y disciplina científica, a lo largo de su historia, ha generado diferentes modelos de práctica profesional, con diversas metodologías y estándares de actuación. A su vez, la realidad social se ha visto sometida a cambios y evoluciones. Por tanto, la aplicación de esos modelos, en una realidad distinta, exige ajustes y reflexiones. Sistematizar los procesos de intervención, como forma fiable de ordenar la práctica y reflexionar sobre ella desde todas las perspectivas posibles, contribuye a ese ajuste y adaptación de los modelos inspiradores y justificadores de la intervención. Concretamente, en la nueva realidad a la que se enfrenta el trabajo social en un campo especifico, como el neurológico, propicia la necesidad de que los modelos resulten eficaces, suficientes y operativos. 
Por ello, planteamos reflexionar y repensar la intervención social que se lleva a cabo con pacientes con ictus para generar nuevas ideas y conocimientos sobre la misma y reinventar otras formas de intervención que posibiliten diferentes procedimientos de actuación que produzcan mayor bienestar, cuando el impacto del ictus en la persona, familia y comunidad origina la intervención de la trabajadora social sanitaria del servicio de neurología.

"El ictus consiste en una alteración brusca en una zona del parénquima encefálico" (Arenillas, 2003: 15-16). No es, a priori, una enfermedad previsible, sino que de una forma inesperada y repentina sobreviene a la persona. En latín significa "golpe", y hace referencia a la brusquedad con que aparecen los síntomas (Plaza, 2003: 5-6).

La American Heart Association- Stroke Outcome Classification (AHA-SOC) que estructura el déficit neurológico en 6 dominios o áreas: cognitiva o del intelecto, emocional, lenguaje o comunicación, motora, sensitiva y visual (Arias, 2009: 26).

\section{Tipología}

Existen fundamentalmente dos tipos de ictus según el tipo de lesión que lo provoca: ictus isquémico e ictus hemorrágico (Plaza, 2007:16).

Por un lado, la isquemia cerebral o ictus isquémico (trombosis, embolia o apoplejía) tiene su origen en la oclusión o taponamiento de un vaso, con lo que la sangre no puede llegar a determinada zona del cerebro. Esa parte queda sin oxígeno y sin los nutrientes que necesita y se lesiona.

El otro tipo de ictus es la hemorragia cerebral o ictus hemorrágico (derrame cerebral o hematoma cerebral). Está ocasionado por la rotura de un vaso dentro del cerebro (hemorragia intracerebral) o en sus envolturas (hemorragia subaracnoidea). Esta sangre no se puede liberar al exterior, provocando también la muerte de los tejidos que están comprimidos (Plaza, 2007: 15-16).

\section{Factores de riesgo}

La enfermedad cerebrovascular está asociada a diversos factores de riesgo. Por un lado, se pueden destacar los factores 
no modificables: principalmente la edad, el sexo, la raza y los antecedentes familiares. Estos factores son inevitables, pero es fundamental identificarlos para que las personas de alto riesgo pueden beneficiarse de controles más rigurosos. Por otro lado, se encuentran los factores de riesgo modificables. Son las principales amenazas en la aparición de un ictus y entre ellos destacan la hipertensión arterial, diabetes, hipercolesterolemia, tabaco y algunas arritmias (Martínez-Vila e Irimia, 2000: 26).

La hipertensión arterial es el factor de riesgo más importante tanto para la isquemia como para la hemorragia cerebral, encontrándose en casi el $70 \%$ de los pacientes con ictus (Martínez-Vila e Irimia, 2000: 27). Los individuos que presentan más de un factor de riesgo cardiovascular son más susceptibles de presentar un evento cerebrovascular que aquellos con un único factor (Moreno et al., 2008: 593).

Los factores de riesgo modificables y no modificables influyen no solo en la aparición del ictus sino en la rehabilitación integral del mismo.

\section{Efectos}

El ictus provoca efectos a todos los niveles. En el plano individual, destacan la preocupación, el temor, la incertidumbre, la inquietud, la impotencia, la angustia y la impaciencia que aparece en los pacientes que le sufren. A nivel familiar, el impacto del ictus provoca, en muchos casos, la no aceptación de la realidad, la desestabilización o la unión familiar, pues no deja indiferente a la familia teniendo que asumir nuevos roles y nueva dinámica familiar (Moltó et al., 2007: 103). El ictus a nivel social, es un problema de salud pública, disminuye las personas activas laboralmente (muchas dejan de trabajar y otras personas tienen que ser reubicadas en otros lugares de trabajo), requiere el establecimiento de mayores apoyos sociosanitarios y los afectados suelen encontrarse con dificultades de adaptación en la sociedad por falta de comunicación o falta de movilidad (Mar et al., 2013).

El $73 \%$ de los casos de dependencia en pacientes mayores de 65 años es consecuencia del accidente cerebrovascular, es uno de los condicionantes más importantes de discapacidad permanente en personas adultas y la segunda causa de demencia tras 
la enfermedad de Alzheimer (Consejo General de Colegios Oficiales de Farmacéuticos, 2015: 15). Además, repercute muy significativamente en la familia y en la comunidad y representa una importante carga económica (Sabater, Berbes y Toca, 2015: 85).

\section{Prevalencia}

En España las enfermedades cerebrovasculares son una causa muy frecuente de morbilidad y hospitalización, constituyendo la segunda causa de mortalidad en la población general, y la primera en mujeres (Brea et al., 2013: 211).

Según la Organización Mundial de la Salud (OMS), la incidencia promedio mundial del ictus se sitúa en 200 casos/100.000 habitantes/año. La incidencia del ictus se incrementa de forma progresiva con cada década de vida a partir de los 55 años, ocurriendo más de la mitad de los casos en pacientes mayores de 75 años (Consejo General de Colegios Oficiales de Farmacéuticos, 2015: 14).

En España, según la encuesta de morbilidad hospitalaria del Instituto Nacional de Estadística (INE) en el año 2016, las estancias hospitalarias por enfermedad cerebrovascular fueron un total de 1.239 .33 , registrándose en Castilla y León un total de 73.746 ingresos.

La tasa de morbilidad hospitalaria en España, según refleja dicha encuesta en el año 2016, es de 227 personas por cada 100.000 habitantes por enfermedad cerebrovascular.

Se prevé un incremento del $27 \%$ en la incidencia del ictus entre los años 2000 y 2025, en relación con el envejecimiento poblacional (Cayuela et. al. 2016: 375).

\section{Perfil del paciente con ictus}

Las enfermedades cardiovasculares constituyen uno de los principales problemas de salud en los países desarrollados (Moreno et al., 2008: 510). No hay un perfil concreto de paciente que sufre ictus, pero existe prevalencia de unos caracteres concretos. Entre estos factores se puede señalar la mayor aparición en personas mayores, siendo la edad media a la que acontece el primer ictus en los varones de 68,8 años y en las mujeres de 74,6 años (Matías-Guiu, et al., 2009: 32). 


\section{Trabajo social sanitario de neurología}

La atención e intervención social con paciente neurológicos $y$, por consiguiente, de los que han sufrido ictus, es competencia del/la trabajador/a social sanitario/a adscrito/a al servicio médico de neurología. Se podría decir, por tanto, que el trabajo social sanitario de neurología, es una especialización del trabajo social sanitario (Olmedo y Arranz, 2017: 41) y requiere de una formación específica acreditada.

El trabajo social sanitario en neurología con pacientes que han sufrido ictus tiene como principales objetivos la detección de problemáticas sociales y el abordaje integral de la situación sociosanitaria del enfermo desde su ingreso hospitalario, estableciendo planes de posthospitalización que faciliten el soporte necesario para seguir las indicaciones terapéuticas y para mantener una calidad de vida óptima tanto en el enfermo como en su principal apoyo, la familia.

Para el desarrollo de estas funciones, el profesional de referencia en dicho servicio tiene que tener conocimientos específicos acreditados, entre otros, sobre el concepto y las secuelas del ictus, terminología clínica, pruebas diagnósticas, código ictus, la estructura y funcionamiento de un servicio clínico de neurología, conocimiento y entendimiento de los distintos profesionales que le conforman, el dominio de protocolos y guías de intervención sobre ictus, además de las competencias concretas en técnicas propias y especificas del trabajo social en el área de neurología, escalas de valoración y fundamentalmente participación en grupos científicos, publicaciones e investigaciones que contribuyan a la evidencia científica de la atención social con pacientes que han sufrido ictus.

\section{METODOLOGIA}

\section{Sistematización de una experiencia}

La sistematización se concibe como la reconstrucción y reflexión analítica sobre una experiencia, interpretando lo sucedido para comprenderlo. Permite, por tanto, obtener conocimientos consistentes y sustentados, comunicarlos, confrontar la experiencia con otras y con el conocimiento teórico existente y así 
contribuir a una acumulación de conocimientos generados desde y para la práctica (Barnechea y Morgan, 2010).

Además, el resultado obtenido aspira a enriquecer, confrontar y modificar el conocimiento teórico, convertirlo en herramienta, entender y transformar nuestras realidades (Cifuentes, 2010).

En este caso, la experiencia a sistematizar es la intervención con pacientes con ictus atendidos en centro hospitalario especializado, Hospital Clínico Universitario de Valladolid, perteneciente a la red pública de la Consejería de Sanidad de Castilla y León.

\section{Objetivos de la sistematización:}

La finalidad de sistematizar esta experiencia radica en la necesidad de que el trabajo social sanitario reflexione sobre las intervenciones que realiza, evitando acciones aisladas sin metodología previa y carentes de fundamento teórico y legal. Esto es necesario para promover avances y mejoras que puedan incorporarse a la práctica profesional del trabajo social sanitario de neurología, un campo por explorar para el profesional de trabajo social sanitario. Por ello, se plantea como objetivo general, obtener aprendizajes y recomendaciones de y para la intervención con pacientes que han sufrido ictus desde el trabajo social sanitario, que permitan la mejora de la intervención y su validación como modelo de intervención.

Los objetivos específicos propuestos para el alcance del objetivo general son los siguientes:

- Objetivo especifico 1: Identificar la especificidad de la intervención del profesional de trabajo social sanitario en el campo de la neurología con pacientes que han sufrido ictus.

- Objetivo específico 2: Determinar los agentes claves en la intervención con pacientes que han sufrido ictus.

- Objetivo específico 3: Dotar de cuerpo teórico a la especificidad del trabajo social sanitario en neurología.

\section{Proceso de sistematización}

El punto de partida para cualquier proceso de sistematización es la participación en la experiencia a sistematizar y la existencia de registros de lo acontecido, como así sucede en este caso. A 
partir de ahí, tomando como referencia a Askunze et al. (2004), el proceso seguido ha sido el siguiente: inicialmente se recupera el desarrollo de la intervención para posteriormente ordenar la información, seguidamente se analiza e interpreta la experiencia para elaborar los aprendizajes y extraer las conclusiones del mismo, finalmente se comunican y socializan los aprendizajes adquiridos en este proceso.

\section{RESULTADOS \\ RECONSTRUYENDO LA EXPERIENCIA}

La intervención social con pacientes que han sufrido ictus sigue un orden, y se puede distinguir diferentes fases de intervención:

\section{Recepción de la demanda y acogida}

La acogida y recepción por parte de la trabajadora social sanitaria de un paciente que ha sufrido ictus puede llegar por varios canales.

De manera general, el caso del paciente que ha sufrido ictus es puesto en conocimiento de la trabajadora social sanitaria del servicio médico de neurología en el momento del ingreso hospitalario, a través de tres posibles vías: médico neurólogo, enfermería, iniciativa propia del paciente o su familia.

\section{Recogida de información y estudio}

La trabajadora social sanitaria del servicio de neurología explora y aborda el conocimiento amplio de la situación socio-familiar (composición familiar, dinámica familiar, nivel de comunicación, incapacidad legal, figura legal de referencia, con qué apoyos reales cuenta, situación laboral, persona/s de contacto, factores psicosociales que inciden en su enfermedad, manifestaciones de problemas sociales sanitarios, cómo gestiona sus emociones en su proceso de enfermedad, entre otros) y recaba información de otras fuentes (atención primaria, servicios sociales, juzgado, lugar de trabajo, tercer sector). 
Para ello, las técnicas e instrumentos utilizados son: entrevistas abiertas y/o semiestructuradas con paciente/familia, observación, genograma, flujograma, reuniones e interconsultas con el equipo del servicio de neurología, interconsultas de coordinación con atención primaria, reuniones con centros de acción social, juzgados, entre otros.

Como soporte documental, se abre a cada paciente su historia social, que queda depositada y custodiada en el despacho de la unidad de trabajo social del hospital.

El estudio y análisis de toda la información recogida permite llegar al diagnóstico social sanitario.

\section{Diagnóstico social sanitario / valoración social}

En esta fase se definen y concretan los factores que inciden en la situación de necesidad/problema psicosocial del paciente y de su entorno (fortalezas y amenazas) y que orientarán el plan de intervención.

La valoración social realizada por la trabajadora social sanitaria de neurología recoge la afectación y extensión de la enfermedad; si existe posibilidad de recuperación, en qué áreas y el tiempo estimado; el conocimiento y la percepción del paciente y la familia ante la aparición del ictus; el tratamiento clínico-social establecido (rehabilitación psicofísica y social que precisa, ayudas técnicas que requiere, apoyos sociales al alta hospitalaria, derivaciones y coordinaciones realizadas, establecimiento de pautas sobre organización y dinámica familiar) y el pronóstico estimado para su recuperación.

Las herramientas de diagnóstico social sanitario que aplica la trabajadora social sanitaria de neurología del Hospital Clínico Universitario de Valladolid, son la escala de Lawton y Brody para conocer la influencia de los factores psicosociales en la aparición de problemas de salud, la escala Gijón para identificar el riesgo social que existe, la escala Zarit para la detección de sobrecarga familiar, la escala Barthel para conocer la valoración funcional de la persona, la entrevista psicosocial individual y familiar, el genograma, la historia de vínculo y las evidencias documentales. Se recogen los códigos diagnósticos de la clasificación internacional CIE -10 (Olmedo y Arranz, 2017: 44). 
El diagnóstico social sanitario equivale, para los/as trabajadores/as sociales sanitarios/as, a lo que el diagnóstico médico significa para los/as médicos. En el diagnóstico, sea médico o social sanitario, se apoya todo el tratamiento (Colom, 2010: 113).

La valoración social integrada dentro del equipo multidisciplinar del servicio de neurología, parte de la discusión y puesta en común de las valoraciones de cada profesional del equipo en función de las áreas afectadas. Se realizan reuniones formales semanales y se favorece el contacto permanente. Se cuenta además con una base de datos informática para la gestión de informes y que ofrece accesos abiertos para personal implicado.

La valoración sociofamilar se registra en el programa informático GACELA. Posteriormente se registrará también el plan de intervención durante el ingreso y el recurso al alta.

\section{Plan de intervención}

El plan de intervención recoge objetivos, áreas, actuaciones a desarrollar y profesionales responsables, en los dos principales momentos: mientras el paciente permanece ingresado, y al alta del mismo, planificando los apoyos necesarios.

La trabajadora social sanitaria forma parte del equipo multidisciplinar y, por tanto, planifica y evalúa conjuntamente con el resto de los componentes del equipo manteniendo cuantas reuniones conjuntas se requieran.

\section{Planificación multidisciplinar del alta hospitalaria}

La trabajadora social sanitaria, como nexo de enlace del sistema sanitario con los distintos sistemas y la comunidad, desarrolla tareas de coordinación interinstitucional (atención primaria, otros especialistas) y de coordinación sociosanitaria, judicial, servicios sociales, laborales, asociaciones de enfermos y familiares.

El objetivo principal es establecer una visión global de las circunstancias y necesidades de salud-enfermedad de la persona que permita identificar y posibilitar el recurso idóneo al alta hospitalaria, para garantizar su recuperación funcional y evitar reingresos hospitalarios. 
Al alta hospitalaria se entrega un informe correspondiente del profesional del trabajo social al paciente para la continuidad de la atención social (Olmedo y Arranz, 2017: 46).

\section{Evaluación y seguimiento}

El seguimiento y la evaluación clínico-social se realiza a los tres meses del alta hospitalaria coincidiendo con la consulta pautada en neurología y a través de citación indicada en el alta. Consiste en una entrevista con el familiar y/o el/la paciente, registrada únicamente en papel y donde se tratan aspectos como: organización y dinámica familiar establecida tras el alta hospitalaria, valoración de la necesidad de otros apoyos, evaluación de las posibilidades de incorporación a la vida laboral, identificación de las dificultades presentes tras el alta hospitalaria, cumplimiento de las recomendaciones tras el alta, entre otras.

\section{DISCUSIÓN}

En España, la figura de los asistentes sociales vinculados a los servicios sanitarios de origen hospitalario, data de los años 60. A modo de ejemplo, en el año 1961 Montserrat Gramut concluía en su tesis:

En los grandes hospitales, el perfeccionamiento técnico y la especialización dificultan las relaciones humanas entre el enfermo y el personal sanitario (...). El Servicio Social Hospitalario consigue perfeccionar su eficacia cuando establece una constante y metódica colaboración entre todas las asistentes sociales que trabajan en instituciones sanitarias. (...) Cabe admitir como indispensable que el asistente social del sector resuma el informe social del cliente y le envíe al asistente social hospitalario. Y a la inversa cuando el enfermo sea dado de alta, los datos obtenidos durante su estancia deben ser enviados al asistente social del sector (Colom, 2008: 66).

Algo más de 10 años después, concretamente en 1977, el extinto Instituto Nacional de Previsión, en la circular 28/1977, definía cuáles eran las funciones de los trabajadores sociales en los hospitales. 
La Organización Mundial de la Salud, en el año 1978, en la declaración de Alma Ata, introduce un cambio en el concepto de salud, no solo como la ausencia de enfermedad, sino como el "bienestar físico, psíquico y social". Este hito histórico, provoca el desarrollo de nuevos modelos en la atención a la salud, acentuando el carácter social de las disciplinas sanitarias, la interdisciplinariedad en las intervenciones en el Sistema de Salud, la coordinación con otras instituciones sociales, siendo la profesión de trabajo social la referencia del ámbito social de la persona en esta nueva concepción de la salud.

En el año 2015, casi cuarenta años después, con la incorporación más intensa (ampliación de tiempo dedicado a este servicio) de la trabajadora social sanitaria del servicio médico de neurología al Hospital Clínico Universitario de Valladolid, detecta la necesidad de la especificidad de la atención social con pacientes que han sufrido ictus, el conocimiento de terminología clínica, las secuelas, signos, síntomas que este produce en el paciente y familia, el gran impacto que produce, precisando intervención a nivel individual, familiar, laboral, social, entre otros. Requiere, por tanto, una respuesta organizada y fundamentada.

La ausencia o escasez de investigaciones e intervenciones científico-empíricas desde el trabajo social sanitario de neurología, publicadas o editadas para el público, sobre una intervención especifica con pacientes a los que les sobreviene una enfermedad como el ictus, asociándola a los beneficios directos e indirectos sobre la persona, su familia y/o su entorno, ha propiciado el interés por analizar esta intervención.

La complejidad a la que se enfrenta el trabajo social sanitario de neurología ante un problema de salud que golpea de manera sobrevenida en el ámbito personal, familiar, laboral, social y en ocasiones, afectación de la capacidad de obrar de la persona, se requiere de una intervención social, y hace por ello necesario el perfeccionamiento a través de estudios, análisis y reflexiones en este ámbito, todavía por descubrir por el trabajo social sanitario.

La sistematización de esta intervención alude a la preocupación de la trabajadora social de neurología del Hospital Clínico Universitario de Valladolid por ahondar y profundizar en este ámbito para garantizar una atención social integral acorde con el impacto que provoca el ictus para dar una respuesta eficaz, 
profesionalizada y avalada con evidencias y conocimientos, alejada de las intervenciones sin metodología, sin estructura ni evidencias. En definitiva, alejarnos de intervenciones basadas en opiniones para lograr intervenciones basadas en evidencias.

\section{ELEMENTOS CLAVE DEL CONTEXTO}

\section{Contexto organizacional}

La Institución donde se desarrolla este trabajo es en el servicio médico de neurología del Hospital Clínico Universitario de Valladolid de Sanidad Castilla y León (SACYL).

La neurología se encarga del estudio, diagnóstico y tratamiento de las enfermedades asociadas a la corteza cerebral y por tanto del ictus.

La unidad de ictus del Hospital Clínico Universitario de Valladolid es un centro terciario, centro de ictus de referencia de las provincias Valladolid, Palencia, Segovia y Zamora, que puede ofrecer el tratamiento para la eliminación del coágulo que ha ocluido la arteria del cerebro, la valoración neurológica urgente y pruebas diagnósticas avanzadas, la monitorización permanente del estado neurológico, de la función cardíaca y respiratoria en una unidad de cuidados semicríticos y el control por personal médico y de enfermería especializado.

\section{Contexto legislativo}

El contexto legislativo que fundamenta la atención social a pacientes neurológicos dentro del sistema de salud de Castilla y León tiene su referencia a nivel nacional en el artículo 43 de la Constitución Española de 1978 sobre el derecho a la protección de la salud y a la atención sanitaria de todos los ciudadanos; en la Ley 14/86 de 25 de abril, General de Sanidad que garantiza el derecho a la salud y le define como el completo estado de bienestar físico, psíquico y social; en la Ley 41/2002, de 14 noviembre, básica reguladora de la autonomía del paciente y derechos y obligaciones en materia información y documentación clínica; en la Ley 16/2003, de 28 de mayo, de cohesión y calidad del Sistema Nacional de salud. 
A nivel regional, destacar, entre otras, la Ley Orgánica 14/2007, 30 noviembre, de reforma del Estatuto de Autonomía de Castilla y León, que en su artículo 13.2 garantiza el derecho a la salud; la Ley $8 / 2003,8$ abril, sobre derechos y deberes de las personas en relación a la salud; el Decreto 49/2003, de 24 abril, por el que se modifica el Decreto 74/2000, de 13 abril, sobre estructuras de coordinación sociosanitaria de Castilla y León y el documento de consenso sobre las funciones del/la trabajador/a social sanitario/a en Castilla y León.

\section{Marco teórico de referencia}

La trabajadora social sanitaria de neurología del Hospital Clínico Universitario de Valladolid, basa su intervención en diferentes modelos de intervención psicosocial.

De forma destacada se puede mencionar el modelo de gestión de caso que tiene como finalidad obtener el tratamiento más apropiado para el usuario, mediante el uso eficiente de los recursos disponibles (Sarabia, 2007: 9).

La Asociación Nacional de Trabajadores Sociales de Estados Unidos (Nacional Asociation of Social Workers) (1992) define la gestión de casos como:

Un método de provisión de servicios en el que un profesional del trabajo social valora las necesidades del cliente, y de su familia cuando sea adecuado, y organiza, coordina, monitoriza, evalúa y apoya (media) un grupo de múltiples servicios para satisfacer las necesidades complejas de un cliente específico (...). La gestión de casos en trabajo social tiene naturaleza tanto micro como macro, y la intervención tiene lugar tanto a escala del cliente como a la del sistema (citado en Argulló et al., 2012: 269).

Otro de los modelos clave es el modelo centrado en la persona, modelo profesionalizado que busca cuidar apoyando que las personas puedan seguir teniendo control en su entorno, en sus cuidados y en su vida cotidiana, desarrollando sus capacidades y sintiéndose mejor. Un modelo profesionalizado que busca aumentar la calidad de la atención desde dimensiones relacionadas con la calidad de vida (Martínez et al. 2015: 3).

Las situaciones de cada persona son muy diferentes, por ello la atención centrada en la persona requiere de una intervención 
interna muy flexible, poco normativizada y que ofrezca oportunidades a cada persona para escoger y elegir qué cosas son para ellas realmente importantes.

El modelo psicosocial es un modelo con influencias freudianas cuyo elemento central es el diagnóstico psicológico de la persona como fundamento de intervención. Mediante este modelo, el/la trabajador/a social busca ayudar al individuo que tiene un problema debido a desórdenes en el funcionamiento intrapsíquico (Arredondo y Cosano, 2013: 139).

Su gran aportación al trabajo social es el descubrimiento de un nuevo paradigma de estudio del ser humano, el cual es contemplado como un organismo bio-psico-social, un todo formado por partes y compuesto por factores tanto internos como externos.

\section{Agentes claves y su influencia}

Debido al carácter integral de la intervención, en el servicio médico de neurología, además del profesional de trabajo social sanitario de neurología, se puede destacar los diferentes profesionales que intervienen con el paciente: especialistas en neurología que diagnostican y tratan la enfermedad; el equipo de enfermería que realiza los principales cuidados y atenciones sanitarias; especialistas en neurorradiología que realizan pruebas radiológicas exploratorias; especialistas en neurocirugía, responsables del área quirúrgica; auxiliares de enfermería que se encargan de las movilizaciones del paciente, aseo y transferencias corporales y otros especialistas que, en interconsulta, se precisan por las necesidades del paciente, como fisioterapeutas, logopedas, entre otros.

Además, se precisa de la colaboración del paciente, del entorno familiar, de la atención social en atención primaria al alta del paciente y de los apoyos del tercer sector específico en patología de ictus y del Sistema Público de Servicios Sociales.

\section{CONCLUSIONES Y APRENDIZAJES: REPENSANDO LA INTERVENCIÓN}

El proceso de sistematización ha identificado una serie de éxitos de la intervención. Entre ellos, se destaca contemplar a 
la persona como un todo, con una atención clínica, social y familiar; el enfoque integral en la recuperación funcional, física y emocional y la atención centrada en la persona potenciando al paciente como sujeto activo de la intervención.

Asimismo, se han visibilizado dificultades, entre ellas, la dificultad de trabajar en equipo para definir áreas afectadas, extensión, límites y acuerdos; interferencias en el proceso cuando intervienen otros profesionales, que enfocan la intervención de diferente manera a la que se había iniciado y acordado; falta de comunicación por la carga asistencial de los profesionales; herramienta informática no adaptada a las intervenciones, valoraciones y/o gestiones realizadas por el profesional del trabajo social.

Los elementos clave para la superación de estas dificultades han sido la concienciación a los profesionales de que las dificultades se pueden superar, la voluntad de los profesionales de superar las adversidades, la promoción de espacios de escucha e intercambio interdisciplinar, el respeto a la disciplina de cada profesional implicado en el tratamiento rehabilitador del paciente y la asunción de la responsabilidad de cada profesional que interviene, entre otros.

El elemento clave principal que potenció la experiencia es el procedimiento interno establecido en el servicio médico de neurología, para poner en conocimiento de la trabajadora social sanitaria el ingreso de un paciente que ha sufrido ictus, de forma que se puedan resolver las incidencias que suceden durante el ingreso. Esto permite concienciar desde el inicio al paciente del cambio de vida que va a suponer el ictus, posibilita el establecimiento de un recurso más adecuado y de forma más planificada y adaptado a sus necesidades reales y contribuye a un mejor acompañamiento social durante el ingreso, facilitando tramitaciones, localizaciones de familiares, acompañamiento hospitalario y/o apoyo espiritual.

Además, como elementos clave se pueden señalar también el contraste de información con atención primaria de salud para agilizar la consecución de evidencias, el análisis integral con los especialistas que intervienen en el tratamiento del paciente y la planificación multidisciplinar del alta hospitalaria. Esta planificación permite una respuesta integral y conjunta a los déficits sufridos en el paciente en todas las áreas: física, psíquica y social; 
facilita al paciente, tempranamente, la adquisición, recuperación y/o el mantenimiento de las capacidades y habilidades personales que favorezcan su desenvolvimiento autónomo el mayor tiempo posible y permite la permanencia en el medio habitual de convivencia y la participación en la vida socio-familiar.

Para finalizar, y fruto del proceso de análisis y reflexión que ha supuesto la sistematización de la intervención, se pueden destacar una serie propuestas de mejora en los procesos de intervención con pacientes con ictus desde el trabajo social.

Es importante la realización de un estudio inicial desde el ingreso de la situación socio familiar del paciente, para evaluar los factores condicionantes que pueden influir positiva o negativamente en su evolución y en su tratamiento, así como el diseño de instrumentos propios y específicos del trabajo social sanitario de neurología que interviene con pacientes que sufren ictus.

Se requiere igualmente establecer canales fluidos y directos de comunicación entre los profesionales; promover el diseño de sistemas de registro único para todos los profesionales, donde cada profesional que interviene en el caso registre en la misma herramienta informática el tratamiento integral del paciente y que permita la inclusión de la valoración realizada en entrevista de seguimiento tras el alta hospitalaria, y fijar el plan de intervención conjunta secuencial, así como el plan al alta hospitalaria, contemplando todas las atenciones que precisa el paciente que sufre ictus.

Además, la intervención ha de contemplar el asesoramiento a pacientes y la tramitación de ayudas económicas para pago de servicios de tratamiento que precise el paciente y no existen en el Hospital Clínico Universitario de Valladolid; el asesoramiento sobre la valoración de la situación de dependencia y la evaluación y el seguimiento continuo que garantice la promoción de mejoras (herramientas de intervención, técnicas, espacios de intercambio de conocimiento interdisciplinar) para el avance de la valoración integral por parte de todos los profesionales que intervienen, evaluando las áreas afectadas del paciente, el tratamiento requerido, estimación del pronóstico o no de recuperación y el apoyo sociofamiliar con el que cuenta. 


\section{REFERENCIAS BIBLIOGRÁFICAS}

Arenillas, J. (2003). Arterosclerosis Intracraneal de gran vaso: progresión y recurrencia clínica. (Tesis para aspirar Grado de Doctor). Universitat Autónoma de Barcelona. Recuperada el día 14 de marzo de 2017. http://www.tdx.cat/bitstream/ handle/10803/4434/jfal1de1.pdf?sequence=1

Arias, A. (2009). Rehabilitación del ACV: evaluación, pronóstico y tratamiento. Galicia Clin; 70 (3): 25-40.

Argulló, A. et al. (2012). Trabajo Social Sanitario y Gestión de Casos. Documentos Trabajo Social Sanitario (51) 268-284.

Arredondo, R. y Cosano, F. (2013). Diccionario Práctico de Trabajo Social. Málaga: Colegio Oficial de Diplomados en Trabajo Social de Málaga.

Askunze, C., Eizaguirre, M. y Urrutia, G. (2004). La sistematización, una nueva mirada a nuestras prácticas. Bilbao: Alboan/ Hegoa/Instituto de Derechos Humanos Pedro Arrupe.

Barnechea, M. M. y Morgan, M. L. (2010). La sistematización de experiencias: producción de conocimientos desde y para la práctica. Tendencias \& Retos (15), 97-107.

Brea, A., Laclausta, M., Martorell, E. y Pedragosa, A. (2013). Epidemiologia de la enfermedad vascular cerebral en España. Clínica e Investigación en Arterioesclerosis, 25 (5), 211-217.

Cayuela, A. et al. (2016). Análisis de las tendencias en la mortalidad por enfermedades cerebrovasculares en España 19802011. Neurología, 31(6), 370-378.

Cifuentes, R. M. (2010). Sistematización de experiencias en Trabajo Social: desafío inminente e inaplazable. III Congreso Internacional Trabajo Social Formación profesional investigación sistematización e identidad profesional en la modernidad, preguntas y respuestas. Arequipa Perú. Recuperado de: http://www.cepalforja.org/sistem/bvirtual/wp-content/ uploads/2016/10/Sistematizaci\%C3\%B3n-de-exps-en-TSdesaf\%C3\%ADo-Inminente-e-inaplazable-RMCG-dic-010.pdf

Colom, D. (2008). El trabajo social sanitario. Atención primaria y atención especializada. Teoría y práctica. Madrid: S.XXI de España Editores. 
Colom, D. (2010). El trabajo social sanitario en el marco de la optimización y sostenibilidad del sistema sanitario. Zerbitzuan (47), 109-119.

Consejo General de Colegios Oficiales de Farmacéuticos (2015). Prevención del Riesgo Cardiovascular. Punto Farmacológico (103),1-38.Recuperadodehttps://www.cofpo.org/tl_files/Docus/ Puntos\%20Farmacologicos \%20CGCOG/20160307\%20 INFORME\%20CONSEJO\%20PREVENCION\%20RIESGO\%20 CARDIOVASCULAR\%2007-03-2016.pdf

Constitución Española. Boletín Oficial del Estado n 311 de 29 de diciembre de 1978.

Decreto 49/2003, de 24 abril, por el que se modifica el Decreto $74 / 2000$, de 13 abril, sobre estructuras de coordinación sociosanitaria de Castilla y León. Boletín Oficial de la Junta de Castilla y León, $n^{\circ}$ 81, de 30 de abril de 2003.

Dirección General de Asistencia Sanitaria de la Gerencia Regional de Salud. (2013) Documento de Consenso sobre las Funciones del profesional de Trabajo Social Sanitario en el Sistema de Salud de Castilla y León. Recuperado el 2 de febrero de 2018 http://www.saludcastillayleon.es/profesionales/es/documentoconsenso-funciones-profesional-trabajo-social-san

INE (2016). Encuesta de morbilidad hospitalaria (EMH).

Instituto Nacional de Previsión (1977). Circular n² 28/77.

Ley 14/1986, de 25 abril, Ley General de Sanidad. Boletin Oficial del Estado n ${ }^{\circ} 102$, de 29 abril de 1986.

Ley 41/2002, de 14 noviembre, básica reguladora de la autonomía del paciente y derechos y obligaciones en materia información y documentación clínico. Boletin Oficial del Estado ${ }^{\circ}$ 274, de 15 de noviembre de 2002.

Ley $8 / 2003$, de 8 abril, sobre derechos y deberes de las personas en relación a la salud. Boletín Oficial del Estado $n^{\circ} 103$, de 30 de abril de 2003.

Ley 16/2003, de 28 de mayo, de cohesión y calidad del Sistema Nacional de salud. Boletin Oficial del Estado n 128, de 29 de mayo de 2003.

Ley Orgánica 14/2007, de 30 noviembre, de reforma del Estatuto de Autonomía de Castilla y León. Boletín Oficial del Estado ${ }^{\circ}$ 288, de 1 de diciembre de 2007. 
Mar, J., etal. (2013). Los costes del ictus en España según su etiología. El protocolo del estudio Conoces, Neurología, 28 (6), 332-339. Recuperadodehttps://ac.els-cdn.com/S0213485312002447/1s2.0-S0213485312002447-main.pdf?_tid=fe79f0001560-11e8-bd98-00000aacb35f\&acdnat $=1519036876$ c28df5dec2989bace3bd04a51b038d9f

Martinez, T., Díaz-Veiga, P., Rodriguez, P. y Sancho, M. (2015). Modelo de Atención Centrada en la Persona. Presentación de Cuadernos prácticos. Madrid, Informes Envejecimiento en Red (2). Recuperado de http:// envejecimiento.csic.es/documentos/documentos/enredmodeloatencioncuadernosmatia.pdf

Martinez-Vila, E., y Irimia, P. (2000). Factores de riesgo del ictus. Anales del sistema sanitario de Navarra (23), 25-31.

Matías-Guiu, J., Villoria, F., Oliva, J., Viñas, S., y Martí, J. (2009). Estrategia en ictus del Sistema Nacional de Salud. Madrid: Ministerio de Sanidad y Política Social.

Moltó, J.M., Lozano, A., Castellví, E. y Villanueva, V. (2007). Las Ayudas. Recursos Sociales tras un Ictus. En Castellvi et al. Guía de información al paciente con ictus (pp.103-107). Valencia: Generalitat Valenciana.

Moreno et al. (2008). Factores de riesgo vascular en pacientes con ictus isquémico. Distribución según edad, sexo y subtipo de ictus. Revista Neurología, 46 (10), 593-598.

Olmedo, V. y Arranz, M. (2017). Trabajo Social Sanitario: Intervención con Pacientes con ictus desde el Servicio Médico de Neurología del Hospital Clínico Universitario de Valladolid. Trabajo Social y Salud (88), 39-47.

OMS (1978). Declaración de Alma Ata. Conferencia Internacional sobre Atención Primaria de Salud Alma-Ata, URSS, 6-12 de septiembre de 1978.

Plaza, I. (2003). Enfermedades cerebrovasculares: Ictus, el azote de la mujer. Summa Neurológica 2 (4), 5-14.

Plaza, I. (2007). ¿Qué es un ictus? En Castellvi, E. et al. Guía de información al paciente con ictus (15-16). Valencia: Generalitat. Conselleria de Sanidad.

Sabater, H., Berbes, L. y Toca, S. (2015). Rehabilitación del ictus. Primera parte. Revista Cubana de Medicina Física 
y Rehabilitación (1), 84-95. Recuperado de http://www. medigraphic.com/pdfs/revcubmedfisreah/cfr-2015/cfr151h.pdf

Sarabia, A. (2007). La gestión de casos como nueva forma de abordaje de la atención a la dependencia funcional. Zerbitzuan (42), 7-17.

Vélez, O. L. (2003). Reconfigurando el Trabajo Social. Perspectivas y tendencias contemporáneas. Buenos Aires: Espacio Editorial. 